\title{
Cáncer de mama con Her-2-neu y receptores hormonales positivos. Tratamiento sistémico primario, biopsia del gan- glio centinela y hormonoterapia
}

\author{
Nayara López $C .^{1}$, José Ignacio Sánchez M. ${ }^{2}$, Javier De Santiago G. ${ }^{1}$ \\ ${ }^{1}$ Servicio de Ginecología, ${ }^{2}$ Sección de Patología Mamaria, Hospital Universitario La Paz. Madrid, España.
}

\section{RESUMEN}

La quimioterapia neoadjuvante aparece como una opción terapéutica interesante en determinados casos de cáncer de mama. En éstos, el momento de la realización de la biopsia del ganglio centinela supone un tema de controversia actual. Los tumores Her-2-neu y receptores estrogénicos positivos presentan cierta resistencia a la terapia hormonal especialmente con tamoxifeno. Se presenta un caso con co-expresión de Her-2-neu y receptores estrogénicos que se trata con quimioterapia neoadjuvante y biopsia de ganglio centinela previa. En un segundo tiempo, se realiza cirugía conservadora sobre la mama con linfadenectomía axilar, radioterapia y hormonoterapia con letrozol, complementada con goserelina y trastuzumab. La evolución posterior ha sido muy favorable.

\section{PALABRAS CLAVE: Cáncer de mama, quimioterapia neoadyuvante, ganglio centinela, terapia hormonal}

\section{SUMMARY}

Neoadjuvant chemotherapy is an interesting option in the therapy of some breast cancer cases. Cases in which the timing for sentinel lymph node biopsy is controversial. Co-expression of estrogen receptors and Her2/neu (c-erbB-2) in breast cancer may imply hormone resistance, especially to tamoxifen. We present a clinic case with co-expression of estrogen receptors and Her2/neu that was treated with neoadjuvant chemotherapy and previous sentinel lymph node biopsy followed by breast tumorectomy with axillar lymphadenectomy, radiotherapy and hormonotherapy with letrozol, geserelina and trastuzumab. A good treatment response was found.

\section{KEY WORDS: Breast cancer, neoadjuvant chemotherapy, sentinel lymph node biopsy, hormonotherapy}

\section{INTRODUCCIÓN}

Actualmente la quimioterapia sistémica primaria se ofrece a pacientes con cáncer de mama en tumores relativamente pequeños $(<3 \mathrm{~cm}$, estadio II). Se ha demostrado que el tratamiento conservador de la mama tras terapia neoadjuvante es seguro en términos de supervivencia y control loco-regional (1).

No hay consenso en la literatura sobre si la biopsia del ganglio centinela debe llevarse a cabo antes o después de la quimioterapia neoadjuvante. Hay autores que consideran que en tumores localmente avanzados no debe realizarse fuera de ensayos clínicos y que debería realizarse siem- 
pre una linfadenectomía (1). Pero, una proporción importante de estas pacientes (40-48\%), no tiene afectación ganglionar (2), por ello son dos las posturas más debatidas. En primer lugar, realizarla después de la quimioterapia minimizando así el número de linfadenectomías en blanco, ante una buena respuesta axilar (3). En el Consenso de Expertos de St. Gallen en 2009, se reconoce su fiabilidad según algunos trabajos. O bien, realizarla antes del tratamiento quimioterápico, con el objetivo de conocer el verdadero estado axilar, y su repercusión en el pronóstico y necesidades terapéuticas (radioterapia axilar sí/no). En estos casos sólo se completaría la linfadenectomía con ganglio centinela previamente positivo (4). Es la postura que defiende la Sociedad Americana de Oncología Médica.

Los tumores que co-expresan receptores hormonales y Her-2-neu pueden presentar resistencia al tratamiento hormonal, principalmente con tamoxifeno, a través de interferencia en las vías de señalización intracelular. La proteína quinasa activada por mitógenos (MAP) cataliza la fosforilación tanto del propio receptor estrogénico como de sus coactivadores provocando un estímulo del efecto proliferativo hormono-dependiente, que no sólo es insensible al tamoxifeno sino que puede ser incrementado por él.

\section{Caso clínico}

Mujer de 32 años que acudió a la Unidad de Patología Mamaria del Hospital Universitario La Paz por palparse un nódulo en mama derecha. En su historia clínica destaca el tratamiento con anticonceptivos orales durante 8 años por Síndrome de Ovario Poliquístico, sin otros antecedentes de interés. En la exploración se palpaba, en la unión de los cuadrantes superiores de la mama derecha, un nódulo de $3 \times 2 \mathrm{~cm}$, indurado, con retracción cutánea, sin adenopatías. La mama izquierda es normal. En la mamografía se apreciaba un nódulo de bordes mal definidos, confirmado por ecografía como nódulo sólido (Figura 1 y 2). La RMN visualizaba un único nódulo y la ecografía axilar era normal. La biopsia con aguja gruesa del nódulo informó de carcinoma ductal infiltrante, con receptores estrogénicos positivos (100\%), receptores de progesterona positivos de forma irregular, p53 positiva, Ki67 50\% y Her-2- neu positivo. El estudio de extensión con TAC toracoabdominal y gammagrafía ósea no mostraron hallazgos relevantes. En el Comité de Tumores de Mama, se decidió iniciar quimioterapia sistémica primaria, tras biopsia de ganglio centinela previa, para posteriormente realizar cirugía, radioterapia y hormonoterapia. El estudio anatomopatológico del único ganglio centinela extraído mostró metástasis de carcinoma de mama con extensión focal extracapsular. Se administraron 6 ciclos de carboplatino-paclitaxel con trastuzumab, con buena tolerancia. En la valoración clínica posquimioterapia, la paciente presentó respuesta parcial. La lesión previa no se delimitaba con claridad en la exploración, pero se apreciaba en la RMN y la ecografía (Figura 3 y 4).

Ante estos hallazgos, se programó una tumorectomía con arpón eco-dirigido y linfadenectomía axilar. El estudio anatomopatológico mostró sólo tejido fibroso mamario, reflejo de la respuesta completa al tratamiento y 10 ganglios linfáticos sin metástasis. Posteriormente se administró radioterapia mamaria, axilar y supraclavicular (50 Gy), se le pautó hormonoterapia con letrozol diario y goserelina mensual durante 5 años, y se completó el tratamiento con trastuzumab durante el primer año. La evolución posterior ha sido favorable, sin recidiva locoregional ni metástasis en 5 años.

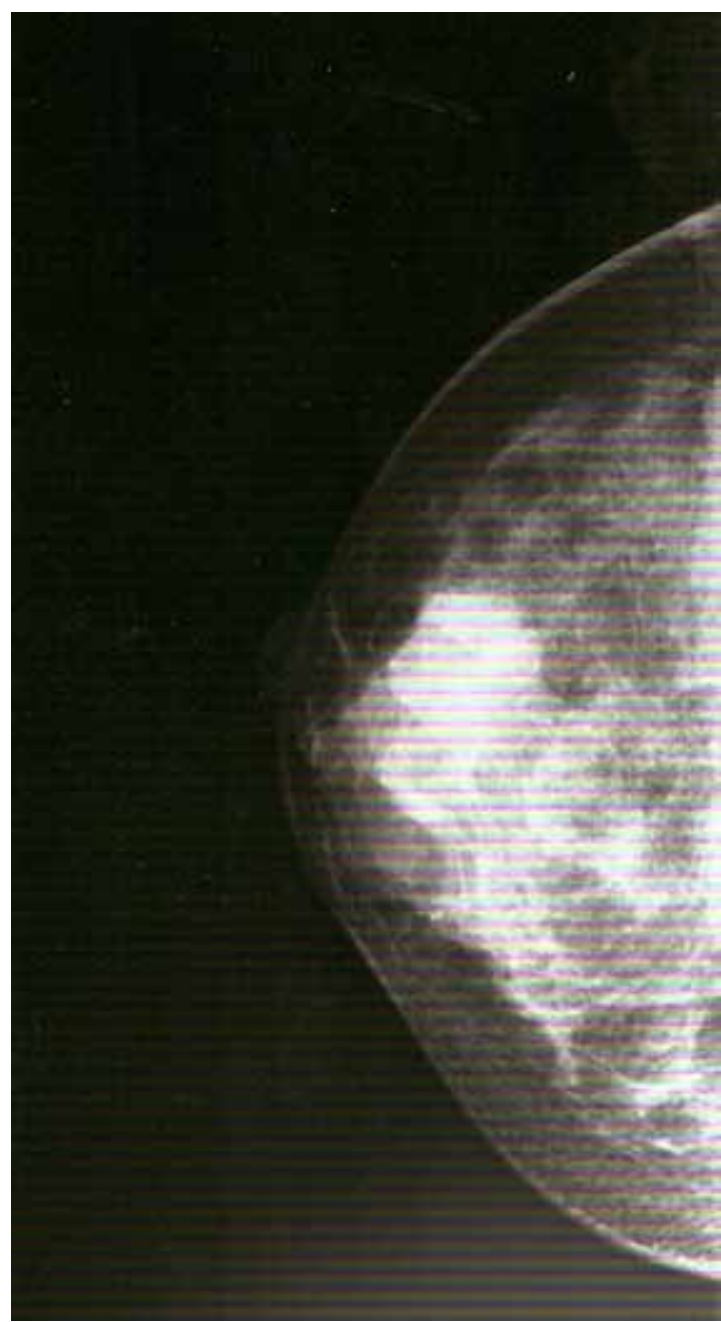

Figura 1. En la mamografía inicial se aprecia un nódulo con tendencia a la espiculación (BIRADS 4). 


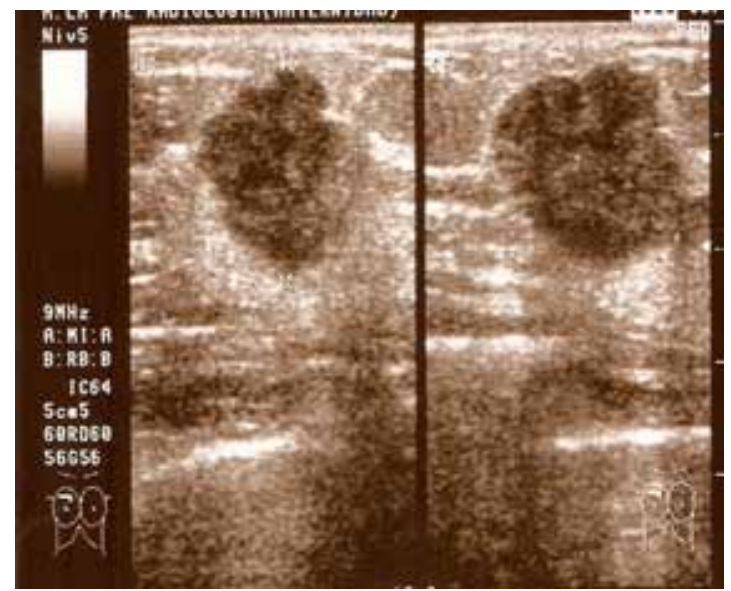

Figura 2. Se visualiza ecográficamente un nódulo mamario lobulado y heterogéneo, que parece invadir el tejido celular subcutáneo, de 24x19,3 mm, en cuadrante supero-externo de mama derecha.

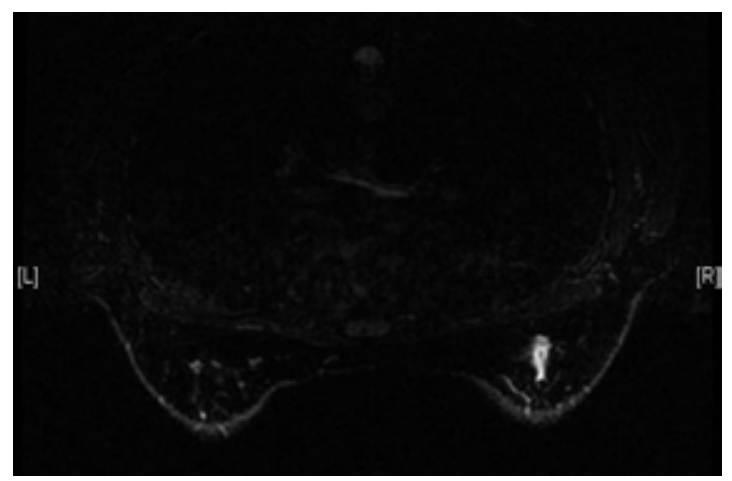

Figura 4. En la RMN se visualiza una lesión nodular en intercuadrantes superiores de mama derecha de 10x15x17 mm, hiperintensa en secuencias TR largo.

\section{DISCUSIÓN}

La opción de tratamiento sistémico primario se planteó en esta paciente con una doble intención. Primero para intentar cirugía conservadora, pues el abordaje quirúrgico inicial no hubiera permitido un buen resultado estético, debido a la relación tumor/ tamaño de la mama, y segundo, para probar la quimiosensibilidad del tumor, lo que a su vez nos sirve como factor pronóstico, puesto que las pacientes con respuesta completa patológica presentan una mejor supervivencia (5). Existen 2 aspectos controvertidos en este caso: el momento de la biopsia del ganglio centinela y la terapia hormonal.

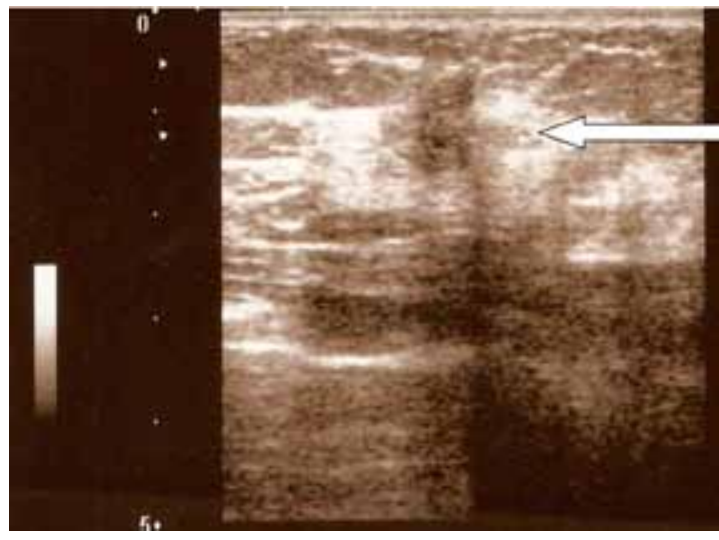

Figura 3. En la ecografía se aprecia un nódulo sólido de unos $10 \mathrm{~mm}$ en cuadrante supero-externo de mama derecha, donde previamente se encontraba el lecho tumoral.

Existe controversia sobre si la biopsia del ganglio centinela debe realizarse antes o después de la administración de la quimioterapia neoadyuvante (1-5). Si se realiza antes, la tasa de éxito del procedimiento puede ser baja, con una alta tasa de falsos negativos (12-15\%) debido a los cambios histológicos producidos en las vías de drenaje mamario por los agentes quimioterápicos (6). La tasa de falsos negativos con la realización del ganglio centinela previamente es equiparable a cuando esta técnica se efectúa de forma conjunta con la extirpación del tumor local. La opción de la realización posterior está basada principalmente en las altas tasas de respuesta mamaria y axilar tras la quimioterapia neoadjuvante, considerando la tasa de falsos negativos asumible especialmente en pacientes que reciben también radioterapia (7). De cualquier modo, resulta imprescindible contar con una buena ecografía axilar.

En esta paciente, una mujer joven, se decidió efectuarla con anterioridad con el objetivo de obtener información sobre el pronóstico y la necesidad de radioterapia axilar posterior, evitando la alta tasa de falsos negativos. En cuanto al tratamiento hormonal, esta paciente co-expresa receptores hormonales y Her-2-neu, hecho poco frecuente en la clínica. En estos tumores existe una relativa resistencia al tratamiento hormonal, especialmente al tamoxifeno, por interferencia en las vías de señalización intracelular. El estímulo proliferativo queda contrarrestado durante la administración de trastuzumab (anticuerpos frente al receptor de Her2-neu), que sólo está indicada durante 1 año. Todo ello plantea serias dudas sobre la conveniencia del empleo de tamoxifeno, al menos a partir del año, y cuál podría ser la alternativa. Los inhibidores de la 
aromatasa tienen un mecanismo de acción diferente, y así existen resultados iniciales en la bibliografía que muestran la superioridad de letrozol frente a tamoxifeno en pacientes con tumores Her-1 y/o Her2 positivos (8). Aunque el tamoxifeno es el agente anti-estrógenos establecido en la terapia hormonal del cáncer de mama, su papel está siendo cada vez más limitado debido a sus efectos colaterales y a su resistencia hormonal en determinados casos de cáncer de mama (9). En las células tumorales que sobreexpresan Her-2-neu y receptores estrogénicos, la terapia combinada con trastuzumab y tamoxifeno es una opción eficaz $(10,11)$. Y la combinación de trastuzumab y fulvestrant es aún más efectiva frente al crecimiento de células que co-expresan Her-2-neu y receptores estrogénicos $(12,13)$.

En el momento actual, sólo está aprobado el uso de inhibidores de la aromatasa en premenopaúsicas en el contexto de ensayos clínicos, excepto si hay contraindicación para el tamoxifeno. A la vista de los resultados mencionados, los tumores que sobreexpresen Her-2-neu y con receptores hormonales positivos, podrían ser considerados al menos una indicación relativa para tratamiento con inhibidores de la aromatasa en premenopaúsicas con tratamiento concomitante con goserelina. Sin embargo, habrá que esperar a tener una base demostrada para su recomendación sistemática.

\section{CONCLUSIONES}

No existe acuerdo sobre el momento de realización de la biopsia de ganglio centinela hasta la existencia de estudios que aporten información sobre recurrencias y pronóstico a largo plazo, por lo que hay que individualizar cada caso. Debido a la acción proliferativa del tamoxifeno en tumores que co-expresan receptores hormonales y Her-2-neu, el tratamiento con letrozol y goserelina trimestral podría ser en el futuro una opción terapéutica en pacientes premenopáusicas.

\section{REFERENCIAS}

1. Schwartz GF, Lange AK, Topham AK. Breast conservation following induction chemotheraphy for locally advanced carcinoma of the breast (stages IIB and III). Surg Oncol Clin 1995;4:657-69.
2. Filippakis G, Zografos G. Contraindications of sentinel lymph node biopsy: Are there any really? Word J Surg Oncol 2007;5:10.

3. Schwartz GF, Tannebaum JE, Jernigan AM, Palazzo JP. Axillary sentinel lymph node biopsy after neoadjuvant chemotherapy for carcinoma of the breast. Cancer 2010;116:1243-51.

4. Mushawah FA, Tan MC, Margenthaler JA. Axillary staging prior to or after neoadjuvant systemic therapy? A single institutional experience. J Surg Oncol 2010;102:404-7.

5. Mauri D, Pavlidis N, loannidos JP. Neoadjuvant versus adjuvant systemic treatment in breast cáncer: a meta-analysis. J Natl Cancer Inst 2005;97:188-94.

6. Charfare $H$, Limongelli $S$, Purushotham AD: Neoadjuvant chemotherapy in breast cancer. $\mathrm{Br} \mathrm{J}$ Surg 2005;92:14-23.

7. Schwartz GF, Giuliano AE, Veronesi U, and the Consensus Conference Group: Proceedings of the Consensus Conference on the Role of Sentinel Lymph Node Biopsy in Carcinoma of the Breast. April 19-22, Philadelphia, PA, USA. Breast J 2002;8:126-38.

8. Ellis MJ, Coop A, Singh B, Mauriac L, Llombert-Cussac $A$, Jänicke $F$, et al. Letrozole is more effective neoadjuvant endocrine therapy than tamoxifen for ErbB1- and/or ErbB-2-positive, estrogen receptor-positive primary breast cancer: evidence from a phase III randomized trial. J Clin Oncol 2001; 19:3808-16.

9. Gago FE, Fanelli MA, Ciocca DR. Co-expression of steroid hormone receptors (estrogen receptor [alpha] and/or progesterone receptors) and Her2/neu (cerbB-2) in breast cancer: Clinical outcome following tamoxifen-based adjuvant therapy. J Steroid Biochem Mole Biol 2006;98:36-40.

10. Witters LM, Kumar R, Chinchilli VM, Lipton A. Enhanced anti-proliferative activity of the combination of tamoxifen plus HER-2-neu antibody. Breast Cancer Res Treat 1997;42:1-5.

11. Kunisue H, Kurebayashi J, Otsuki T, Tang CK, Kurosumi M, Yamamoto S, et al. Anti-HER2 antibody enhances the growth inhibitory effect of anti-oestrogen on breast cancer cells expressing both oestrogen receptors and HER2. Br J Cancer 1999;82:46-51.

12. Pietras RJ. Interactions between estrogen and growth factor receptors in human breast cancers and the tumor-associated vasculature. Breast J 2003;9:361-73.

13. Abdulkareem IH, Zurmi IB. Review of hormonal treatment of breast cancer. Niger $\mathrm{J}$ Clin Pract 2012;15:9-14. 